\title{
Plasmid-encoded NP73-102 modulates atrial natriuretic peptide receptor signaling and plays a critical role in inducing tolerogenic dendritic cells
}

\author{
Weidong Zhang ${ }^{1 \dagger}$, Xueqin $\mathrm{CaO}^{2 \dagger}$, Dongqing Chen ${ }^{1}$, Jia-wang Wang ${ }^{3}$, Hong Yang ${ }^{4}$, Wenshi Wang ${ }^{5}$, \\ Subhra Mohapatra ${ }^{2,6}$, Gary Hellermann ${ }^{1}$, Xiaoyuan Kong ${ }^{1}$, Richard F Lockey ${ }^{1,3}$, Shyam S Mohapatra ${ }^{1,3,6^{*}}$
}

\begin{abstract}
Background: Atrial natriuretic peptide (ANP) is an important endogenous hormone that controls inflammation and immunity by acting on dendritic cells (DCs); however, the mechanism remains unclear.

Objective: We analyzed the downstream signaling events resulting from the binding of ANP to its receptor, NPRA, and sought to determine what aspects of this signaling modulate DC function.

Methods: We utilized the inhibitory peptide, NP73-102, to block NPRA signaling in human monocyte-derived DCs ( $\mathrm{hmDCs}$ ) and examined the effect on DC maturation and induced immune responses. The potential downstream molecules and interactions among these molecules involved in NPRA signaling were identified by immunoprecipitation and immunoblotting. Changes in T cell phenotype and function were determined by flow cytometry and BrdU proliferation ELISA. To determine if adoptively transferred DCs could alter the in vivo immune response, bone marrow-derived DCs from wild-type C57BL/6 mice were incubated with ovalbumin (OVA) and injected i.v. into C57BL/6 NPRA-/- knockout mice sensitized and challenged with OVA. Lung sections were stained and examined for inflammation and cytokines were measured in bronchoalveolar lavage fluid collected from parallel groups of mice.
\end{abstract}

Results: Inhibition of NPRA signaling in DCs primes them to induce regulatory T cells. Adoptive transfer of wild type DCs into NPRA-/- mice reverses the attenuation of lung inflammation seen in the NPRA-knockout model. NPRA is associated with TLR-2, SOCS3 and STAT3, and inhibiting NPRA alters expression of IL-6, IL-10 and TGF- $\beta$, but not IL-12.

Conclusions: Modulation of NPRA signaling in DCs leads to immune tolerance and TLR2 and SOCS3 are involved in this induction.

\section{Introduction}

Allergic asthma is a chronic inflammatory disease of the lung, involving an aberrant T helper-2 (Th2) immune response to allergens. The etiology of asthma is complex and involves a number of signaling molecules and pathways as well as environmental factors. Atrial natriuretic peptide (ANP) is a cardiac hormone that regulates blood pressure and volume and the sodium/potassium

\footnotetext{
* Correspondence: smohapat@health.usf.edu

† Contributed equally

'Department of Internal Medicine, Division of Allergy and Immunology, University of South Florida College of Medicine, Tampa, FL 33612, USA Full list of author information is available at the end of the article
}

balance. Atrial natriuretic factor is synthesized as a prohormone that is cleaved into a C-terminal peptide, ANP, and a group of three $\mathrm{N}$-terminal peptides which are released into the circulation and may negatively inhibit ANP activity. ANP binds to a cell surface receptor, natriuretic peptide receptor A (NPRA), which is found on cells in the lung and airways as well as kidney and other tissues. Hormone binding to NPRA is the predominant trigger in the natriuretic system and generates the intracellular signaling molecule cyclic guanosine monophosphate (cGMP) which can activate cGMPdependent protein kinase and initiate a cascade of events. Patients with asthma or inflammatory lung 
disease have elevated levels of circulating ANP [1], which suggests that manipulation of NPRA signaling might provide a therapeutic benefit for asthmatics [2].

The natriuretic peptide family comprises atrial natriuretic peptide, ANP, brain natriuretic peptide, BNP, C-type natriuretic peptide, CNP, Dendroaspis natriuretic peptide, DNP, and urodilatin [3]. The activities of ANP and BNP are similar, and their biological actions, such as vasodilation and natriuresis, are mediated through binding to their receptor, NPRA, which leads to production of the intracellular second messenger cGMP [4]. Besides expression in heart atria, ANP is also produced in various lymphoid organs [5], and the NPRA receptor is found on immune cells of numerous species highlighting the importance of NPRA signaling in the immune response [6].

Over-production of ANP can affect the adaptive immune system by altering dendritic cell (DC) differentiation and promoting a Th2 response characteristic of allergic disease [7]. However, the mechanism by which NPRA signaling in DCs alters the innate and adaptive immune responses is unclear. In animal models of allergic lung inflammation, we showed that ANP signaling through NPRA promotes lung histopathology [8]. In studying the regulation of NPRA, we discovered a peptide, NP73-102, from the N-terminus of the ANP prohormone that acts as a brake on ANP signaling by reducing expression of NPRA. NP73-102 consists of amino acids 73 to 102 of the ANP prohormone and has bronchoprotective effects in a mouse model of asthma and anti-inflammatory activity in human epithelial cells [9]. The amino acid sequence of this peptide is different from ANP and NP73-102 does not bind to NPRA and prevent ANP from attaching. NP73-102 reduces ANPinduced signaling by downregulating its receptor and by feedback inhibition of ANP production [10].

DCs express abundant NPRA while macrophages do not. It was therefore hypothesized that the effects of NPRA signaling on innate and adaptive immunity occur through NPRA-mediated alterations in gene expression in DCs. Little is known about the role of NPRA signaling in innate immunity and about the downstream effects of NPRA-mediated immunoregulation in DCs. Tolerogenic DCs present antigen to T cells, but do not deliver the signals for effector T-cell activation and proliferation. This lack of costimulation can result in T-cell apoptosis [11], T-cell anergy [12] or differentiation into regulatory T-cells (Tregs) [13]. The identification of ANP's effects on DCs as key regulators of peripheral tolerance to allergens may be important in the prevention and treatment of allergic diseases [14,15].

In this study, the NPRA inhibitory peptide NP73-102 was utilized to block NPRA signaling in human DCs and to analyze the downstream cascade events. The results demonstrate that Toll-like receptor-2 (TLR2) and suppressor of cytokine synthesis-3 (SOCS3) are key players in integrating NPRA signaling with innate immunity and in the induction of tolerogenic DCs.

\section{Materials and methods \\ Isolation, transfection and viability assay of human dendritic cells}

Human monocytes were isolated from peripheral blood mononuclear cells using the Monocyte Isolation Kit II (Miltenyi Biotec, Auburn, CA). Human monocytederived DCs (HmDCs) were generated from these cells as previously described [16]. Briefly, monocytes isolated from peripheral blood mononuclear cells were induced to differentiate into DCs by incubation with $200 \mathrm{ng} / \mathrm{ml}$ IL-4 and $50 \mathrm{ng} / \mathrm{ml} \mathrm{GM-CSF}$. Five day-old DCs were transfected with the indicated plasmid $\left(3 \mu \mathrm{g} / 10^{6}\right.$ cells) using Lipofectamine 2000 (Invitrogen, Carlsbad, CA). After $24 \mathrm{hr}$, cell viability was measured by MTT assay (Sigma/Aldrich, St Louis, MO). Additional information on the viability assay is provided in Additional File 1.

\section{Cytokine measurement}

Transfected hmDCs $\left(1 \times 10^{6}\right.$ cells/well $)$ were cultured in 24-well plates for $24 \mathrm{~h}$. Cytokine levels in the cellculture supernatants were measured using a cytokine bead array kit (BioSystem, Bio-Rad) following the manufacturer's directions. All samples were assayed in duplicate.

\section{Isolation of naïve T cells, generation of Tregs and T-cell suppression assay}

Human allogeneic naïve CD4+CD25-T cells were purified from umbilical cord blood using the naïve $\mathrm{T}$ cell isolation kit with biotinylated CD25 antibody (Miltenyi Biotec, Auburn, CA) and co-cultured with irradiated transfected hmDCs (10:1 ratio) in 24-well plates for 6 days. Expression of the Treg protein FoxP3 by cocultured $\mathrm{T}$ cells was quantitated by flow cytometry (BDFACScan, BD Biosciences, San Jose, CA). Also, total RNA was extracted and analyzed by reverse transcriptase-PCR for FoxP3. Tregs were purified with the Treg cell isolation kit (Miltenyi Biotec.) and a co-culture suppression assay was performed using a BrdU proliferation ELISA kit (Roche, IN, USA) as previously described [17]. Additional information is provided in the Additional File.

\section{Measurement of intracellular cGMP}

Five day-old hmDCs $\left(10^{6}\right.$ cells/sample) were transfected with the indicated plasmid and incubated in medium for $18 \mathrm{~h}$. After incubation, the cells were removed from the plate, pelleted by centrifugation $(750 \times \mathrm{g}, 5 \mathrm{~min})$ and intracellular cGMP in the cell pellets was measured with a cGMP ELISA kit (R \& D Systems, Minneapolis, MN). 


\section{Phagocytosis assay}

Five day-old hmDCs $\left(10^{6}\right)$ were harvested $24 \mathrm{hr}$ posttransfection and resuspended in RPMI 1640 medium supplemented with $2 \%$ FBS. The FITC-dextran phagocytosis assay was performed as described [18].

\section{Immunoprecipitation and immunoblotting}

Five day-old hmDCs were transfected with $3 \mu \mathrm{g}$ each of expression plasmid encoding NPRA, TLR2, STAT3 and SOCS3 and then harvested $24 \mathrm{hr}$ post-transfection. Lysates $(400 \mu \mathrm{g}$ of protein/sample) were immunoprecipitated with antibody against TLR2, STAT3, SOCS3 or NPRA overnight at $4^{\circ} \mathrm{C}$. The antibody complexes were precipitated by the addition of recombinant protein $G$ agarose (Invitrogen, Carlsbad, CA). Eluted proteins were resolved on $12 \%$ SDS-PAGE gels, transferred to PVDF membranes (Bio-Rad, Hercules, CA) and immunoblotted with the indicated antibodies.

\section{Luciferase assay}

HmDCs were transfected with the indicated plasmid and $48 \mathrm{~h}$ later, the cells were analyzed for luciferase activity. Additional detail is provided in the Additional File.

\section{Studies in mice: OVA sensitization, DC isolation and adoptive transfer}

Bone marrow cells were removed from C57BL/6 NPRA-/or wild type (WT) mice and cultured for 8 days as described previously [19]. Bone marrow-derived DCs (bmDCs) were purified using CD11c microbeads (Miltenyi Biotec, Auburn, CA), incubated with ovalbumin (OVA; $0.5 \mathrm{mg} / \mathrm{ml}$ ) for $24 \mathrm{hr}$ and injected i.v. into NPRA-/- mice $\left(5 \times 10^{6} \mathrm{bmDCs} / \mathrm{mouse}\right)$, which had been sensitized (i.p) and challenged (i.n) with OVA $(25 \mu \mathrm{g})$. Mice were euthanatized, lungs were lavaged with $1 \mathrm{ml}$ of $\mathrm{PBS}$, and BAL cytokines were quantitated by cytokine bead array (BioSystem, Bio-Rad, Hercules, CA). Lung histopathology was assessed using a previously described scoring system [20]. Additional details are provided in the Additional File.

\section{Statistical analysis}

The results are expressed as means \pm SEM. Data were analyzed using an unpaired two-tailed Student's $t$ test.

\section{Results}

NP73-102 inhibits NPRA signaling in human DCs

Both endogenous ANP and NPRA are expressed by cultured hmDCs (Figure 1A). Transfection of hmDCs with pNP73-102, however, decreases the expression of endogenous ANP and NPRA compared to controls (Figure 1A). To determine if NP73-102 overexpression caused cytotoxicity in DCs, cell viability was measured $24 \mathrm{hr}$ post-transfection. NP73-102 did not significantly affect cell viability at the dose used (Figure 1B). Intracellular
cGMP levels in transfected hmDCs were also measured at $18 \mathrm{hr}$ post-transfection. NP73-102 dose-dependently decreased cGMP production by blocking the activity of endogenous ANP compared to controls (Figure 1C), indicating that NP73-102 inhibits NPRA signaling in human DCs. The transfection efficiency of hmDCs transfected with pEGFP, as assessed by fluorescence microscopy of green fluorescent protein, was $41.3 \%$.

\section{Inhibiting NPRA signaling alters cytokine production in human DCs}

As demonstrated in Figure $1 \mathrm{~A}$, hmDCs produce endogenous ANP that can be down-regulated by NP73-102. Activation of the ANP-NPRA signaling pathway can alter cytokine profiles in hmDCs and inhibiting this pathway with pNP73-102 allowed greater production of IL-6, IL-10 and TGF- $\beta$ (Figure 2A \&2B, E \&2F). IL-12 and IFN- $\gamma$ levels did not change significantly among the groups (Figure 2C \&2D).

\section{Inhibiting NPRA signaling in human DCs induces Tregs}

Since blocking NPRA signaling by transfection of hmDCs with pNP73-102 up-regulates IL-10 and TGF- $\beta$ expression, the role of NPRA in Treg induction was investigated. HmDCs were transfected with pNP73-102, pANP or pVAX and cocultured with allogeneic naïve CD4+ CD25-T cells (FoxP3-negative by RT-PCR). NP73-102 induced more FoxP3 expression than did ANP or vector alone (Figure 3A \&3B). NP73-102induced CD25+ T cells were able to suppress proliferation of CD4+CD25-T-cells in a dose-dependent manner (Figure 3C), suggesting that CD4+CD25+ T cells with suppressive ability can arise from CD4+CD25-naïve $\mathrm{T}$ cells co-cultured with NP73-102-treated hmDCs, correlating with the expression of FoxP3.

\section{Inhibiting NPRA signaling does not affect maturation of human DCs}

The degree of hmDC maturation affects their capacity for Treg generation, and therefore the NPRA signal blockade was investigated to see if it alters hmDC maturation. Using the ability to phagocytose as a measure of maturation, pVAX or mock transfection slightly lowered hmDC phagocytosis compared to pNP73-102 or pANP (Figure 3D). Further, the hmDC phenotype analysis showed that HLA-DR, CD11c, CD40 and CD80 did not significantly change in expression among the groups (Figure 3E), suggesting that NPRA signaling does not affect hmDC maturation.

\section{NPRA inhibition alters expression of TLR-2, STAT3 and SOCS3 in human DCs}

IL-6 operates through the JAK1-STAT3 pathway, and triggering TLRs on mouse DCs can induce SOCS1 


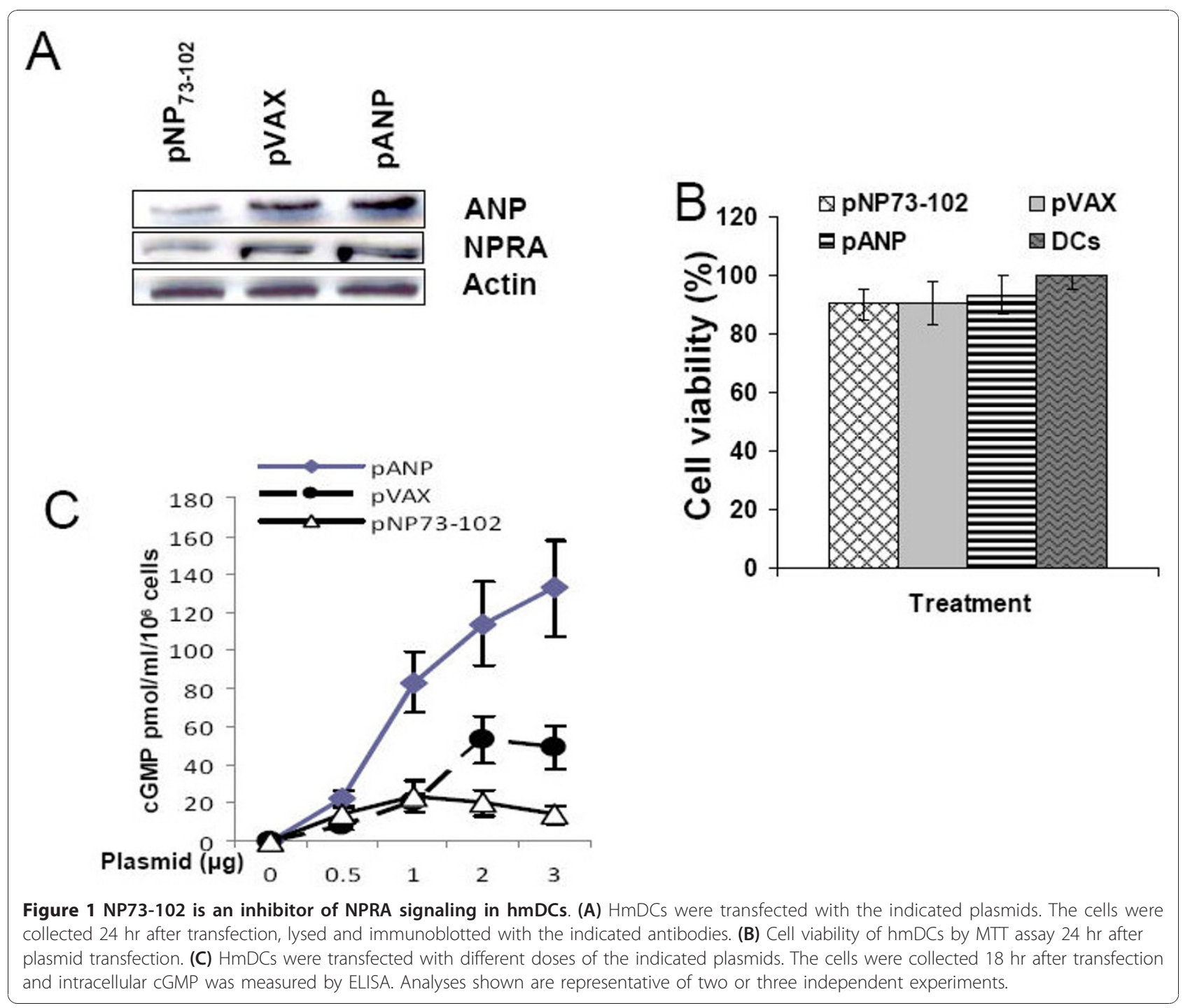

and SOCS3 in a STAT-dependent manner [21]. Results show that transfection with pNP73-102 down-regulated the level of activated phospho-STAT3 protein and enhanced expression of SOCS3 (Figure 4A), but not SOCS1 (data not shown). pNP73-102 selectively decreased TLR2 expression on DCs while elevating MyD88 compared to pANP or pVAX. We also found that pNP73-102 reduced the NF- $\kappa \mathrm{B}$ transactivation from a promoter construct in hmDCs (Figure 4A). To confirm this effect, a luciferase reporter system was utilized to test the effect of NPRA inhibition on promoter activity of some target genes. As shown in Figure 4B-D, NP73-102 attenuated the activity of STAT3/NF- $\kappa \mathrm{B}$ and increased the activity of SOCS3 in hmDCs.

\section{Protein interactions in the NPRA signal pathway}

This set of experiments was designed to determine which of the proteins that are known to be involved in DC function might be associated with NPRA. The hmDCs were transfected with NPRA, TLR2, STAT3 and SOCS3 expression plasmids, allowed to express for $24 \mathrm{hr}$, then whole-cell lysates were immunoprecipitated with the indicated antibody. The levels of expression of each of the proteins in the transfected cells were approximately the same as shown by the second set of bands in each IP. Precipitates were recovered and eluted proteins were separated by SDS-PAGE and immunoblotted for the indicated protein (first set of bands). The blots showed that NPRA was strongly pulled down with TLR2 and STAT3 and weakly with SOCS3 (Figure 5A). The corresponding bidirectional pull-down assays were done and showed similar results (data not shown). TLR2 was bound to STAT3, and the adaptor protein MyD88 bound both STAT3 and SOCS3 (Figure 5A). The IP data suggest that the immunoregulation of NPRA signaling in hmDCs may involve a specific interaction among these four proteins 

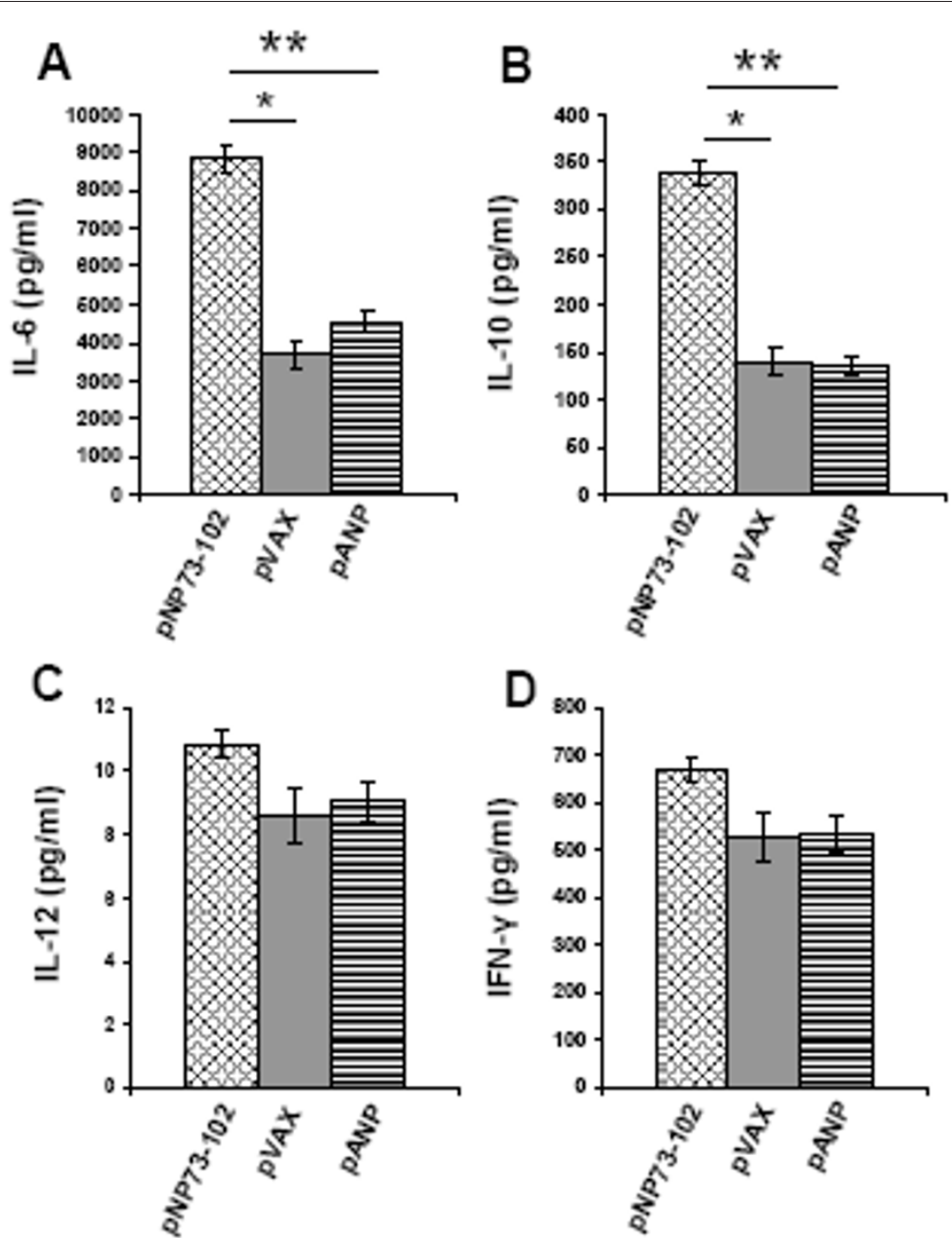

E

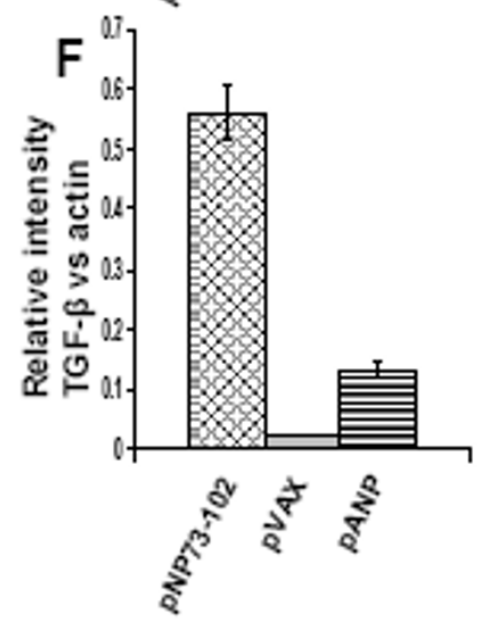

Figure 2 NP73-102 inhibition of NPRA signaling alters hmDC cytokine profile. (A-D) Transfected hmDCs were cultured in complete medium in 24-well plates for $24 \mathrm{hr}$. Cytokine levels in supernatants were measured in duplicate by cytokine bead array assay. The values are means \pm SEM. ${ }^{*} p<0.05$ and ${ }^{* *} p<0.05$ for NP73-102 vs pVAX and pANP respectively. (E) Immunoblotting for TGF- $\beta$ expression in transfected DCs. (F) Protein bands were scanned and band density was quantitated using the Scion Image program. These results are from three separate experiments. 
A

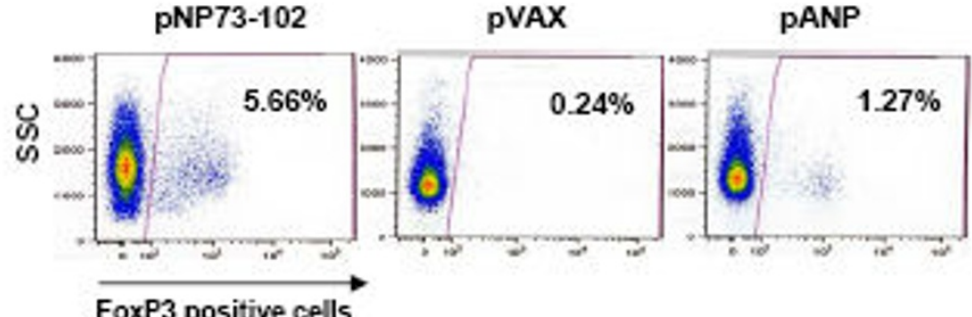

B

Fositive cells

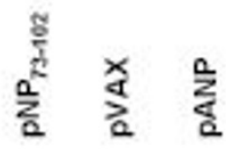

D

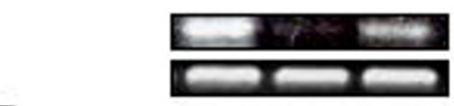

Foxp3

GAPDH
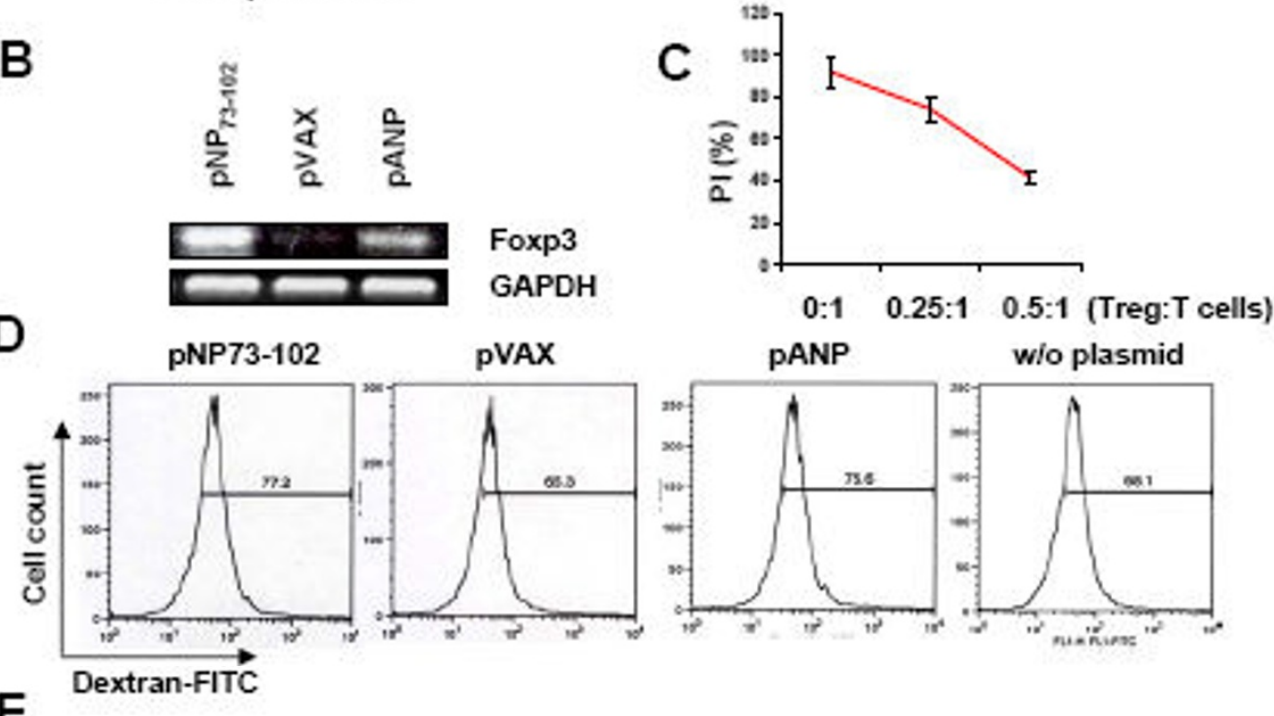

E
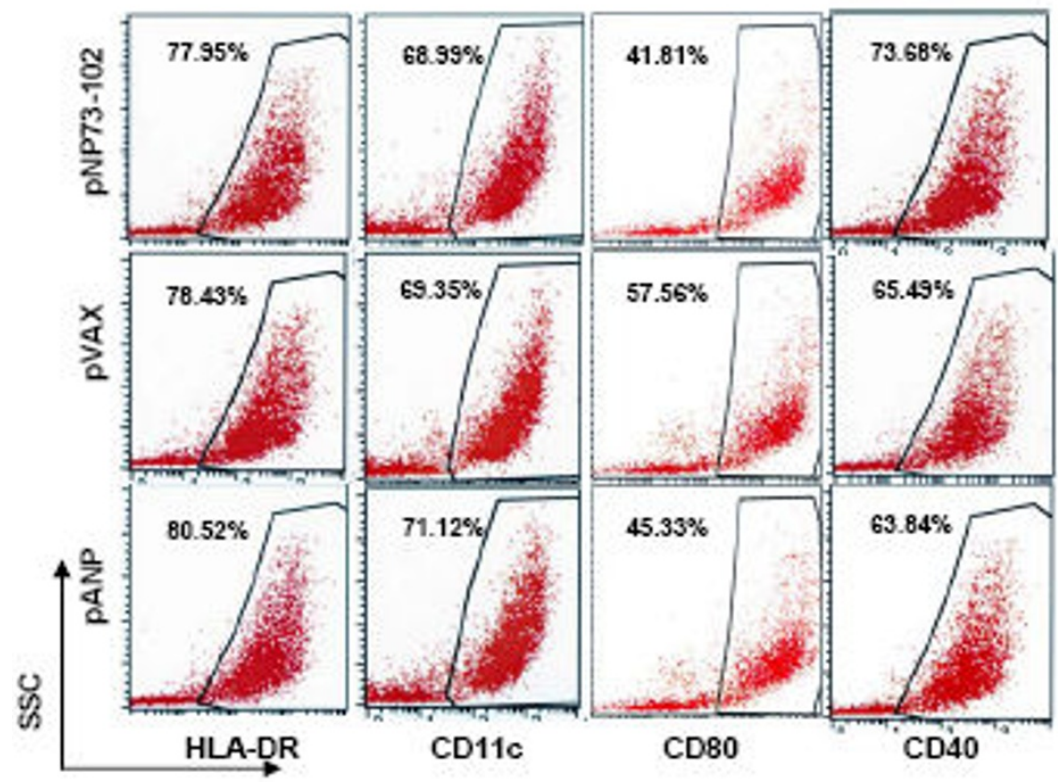

Figure 3 Modulation of NPRA signaling in human monocyte-derived dendritic cells (hmDCs) alters Treg generation in vitro. (A) Flow cytometry assay for Foxp3-positive T cells in naïve CD4+ CD25-T cells co-cultured with plasmid-transfected hmDCs. Results shown are from one representative experiment of three repeats. (B) RT-PCR analysis of Foxp3 expression in naïve CD4+ T cells after co-culture with plasmidtransfected hmDCs. (C) Autologous CD4+CD25-T cells were co-cultured with NP73-102-induced CD4+CD25+ T cells at different ratios and the proliferation index (PI) was calculated. (D) Phagocytosis by hmDCs transfected with the various plasmids. DCs were harvested one day after transfection, incubated with FITC-dextran for $1 \mathrm{~h}$ at $37^{\circ} \mathrm{C}$ and counted by flow cytometry. For each sample, the background (mean value of fluorescence of cells exposed to FITC-dextran at $4^{\circ} \mathrm{C}$ ) was subtracted from the mean value of fluorescence of hmDCs incubated at $37^{\circ} \mathrm{C}$. (E) Flow cytometric analysis of phenotypic markers of transfected hmDCs. Results shown are from one representative experiment of three repeats. 


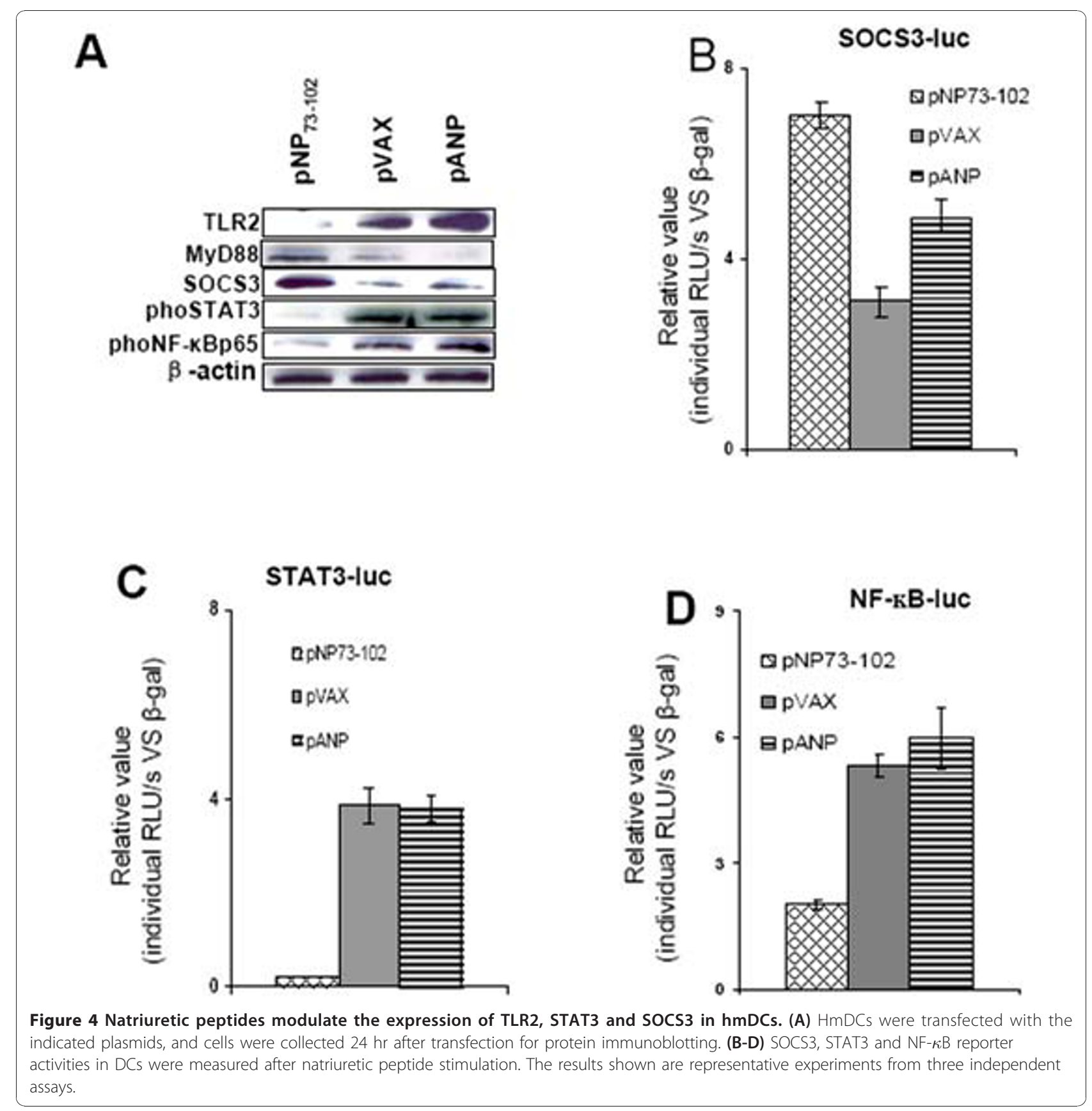

(Figure 5B). The tentative model shows one possible configuration that fits the observed association data, but further work is needed to clarify whether ANP binding to NPRA influences TLR2 activation or vice versa and how NP73-102 might alter the protein interactions.

Adoptively transferred wild type mouse bone marrowderived DCs (bmDCs) restore lung inflammation in NPRA-/- mice

Since hmDCs express NPRA [7] and NP73-102 prevents $\mathrm{NF}-\kappa \mathrm{B}$ activation in hmDCs in vitro (Figure $4 \mathrm{~A}, \mathrm{D}$ ), we reasoned that alterations in NPRA signaling in DCs should affect inflammation in vivo. To test this hypothesis, OVA-treated bmDCs from NPRA-/- or WT C57BL/6 were adoptively transferred into NPRA-/- mice that had been sensitized and challenged with OVA. As shown in Figure 6A, the lungs from NPRA-/- mice given bmDCs from wild-type mice exhibited inflammation similar to that of WT; however, OVA-treated NPRA-/ - mice given no bmDCs or given bmDCs from NPRA-/- mice had little lung inflammation, suggesting that NPRA signaling in DCs plays a critical role in allergic 


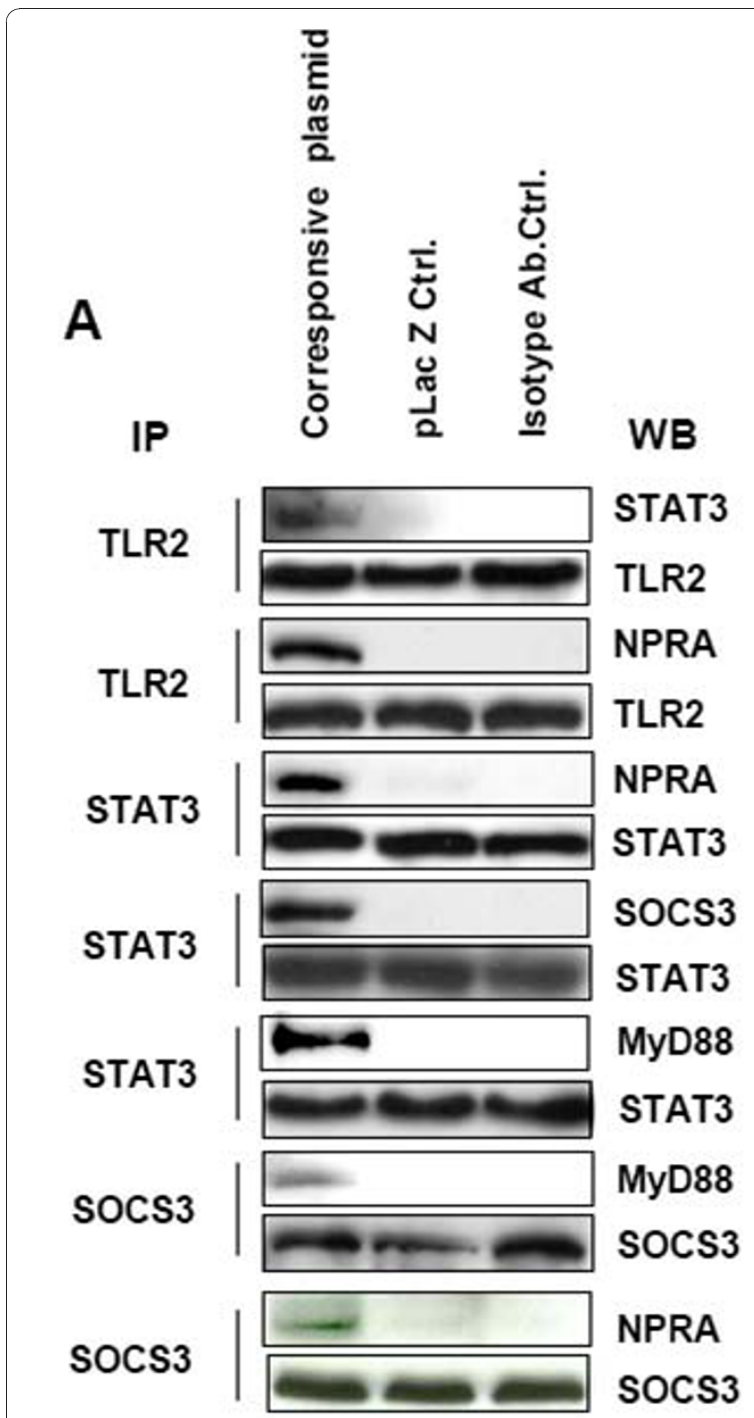

Figure 5 Protein interaction analysis. (A) HmDCs were harvested one day after transfection with the indicated plasmid. Cell lysates were immunoprecipitated (IP) with antibody against TLR2, STAT3, SOCS3 or NPRA and immunoblotted (IB) with STAT3, SOCS, MyD88, or NPRA antibodies, respectively. A representative experiment from three independent assays is shown. (B) Diagram of hypothesized protein interactions in the immune response associated with NPRA signaling in hmDCs.

lung inflammation. Inflammatory scoring by blinded observers of lung sections under the microscope (Figure 4B) confirmed the visualized results of Figure 4A. Cytokines were measured in BAL fluid collected from parallel groups of mice, and the results show that NPRA-/- mice given no bmDCs or given bmDCs from NPRA-/- mice have decreased expression of IL-4, TNF- $\alpha$ and RANTES, and increased IL-10 expression compared to other groups (Figure 6C).

\section{Discussion}

A novel mechanism for inducing tolerogenic DCs by inhibition of NPRA signaling in DCs is described. Our demonstration that down-regulation of NPRA levels and

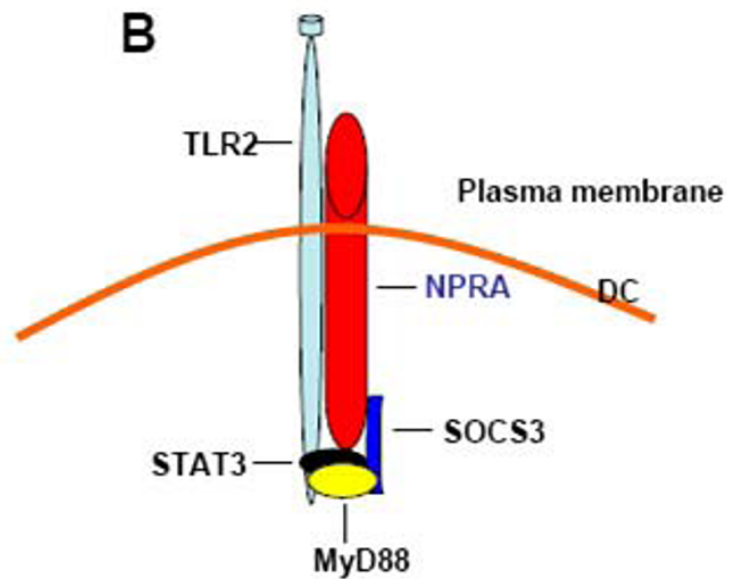

reduction in NPRA signaling in DCs increases the population of Tregs may have important applications in treating respiratory disease and inflammatory conditions. Effective allergen immunotherapy involves generation of Treg cells, and targeted NPRA down-regulation may be used as a means to develop tolerogenic DCs that induce Tregs to ameliorate or prevent inflammation.

The discovery of the NPRA inhibitory peptide NP73102, which reduces expression of NPRA and inhibits NPRA signaling and the activation of several pro-inflammatory transcription factors in epithelial cells $[8,9]$, has provided the impetus to study the mechanism of how NPRA signaling affects inflammation and immunity. Increased NPRA signaling in DCs leads to a Th2 response, 


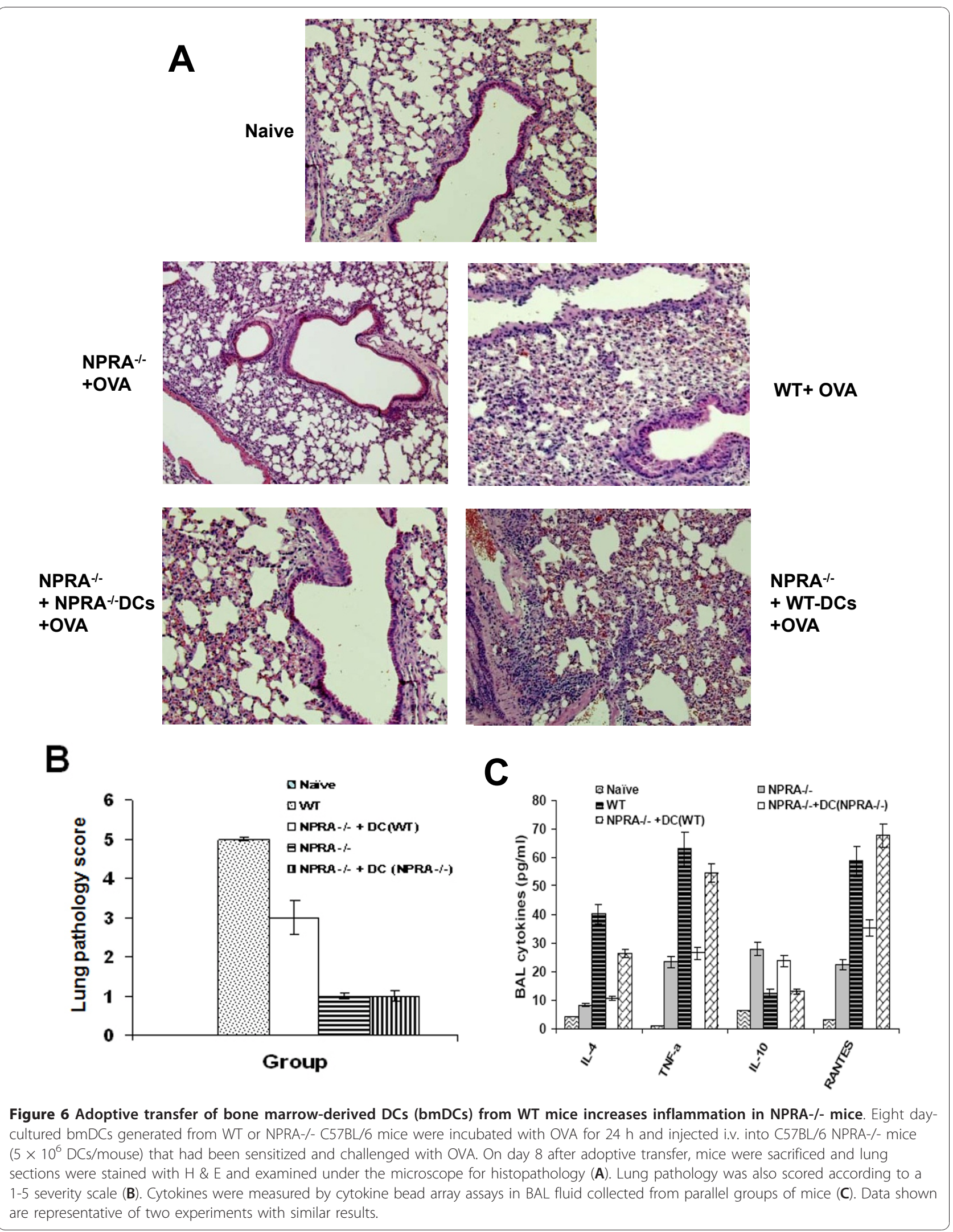


which restricts Treg production, while inhibiting signaling induces more IL-10 and TGF- $\beta$ production and stimulates Treg formation.

Using an in vitro $\mathrm{T}$ cell-DC coculture system, downregulation of NPRA by NP73-102 resulted in greater DC-mediated generation of Tregs. Analysis of DC surface marker expression and in vitro phagocytosis demonstrated that DC maturation was not significantly affected by NPRA signaling blockade. The mechanism underlying the NP73-102-induced Treg response is not fully understood. It may be due to inactivation of NF- $\kappa$ B activity by NP73-102 inhibition of NPRA signaling, since inhibition of NF- $\kappa$ B in DCs enhances their tolerogenic activity and prevents detrimental autoimmune diseases [22].

These data demonstrate that pNP73-102 increases the level of IL-10 and TGF- $\beta$, but not of IL- 12 and IFN- $\gamma$, compared to cells given pANP or vector alone. TGF- $\beta$, which inhibits Th1 and Th2 development, is critical in Th17 development, in combination with IL-6 [23] and leads to the generation of Foxp3-positive regulatory $\mathrm{T}$ cells [24]. However, there was no increase in the number of Th17 lymphocytes, even in the presence of IL-6, in the DC-naive $\mathrm{T}$ cell co-culture system, suggesting that Tregs may inhibit Th17 generation or that different cytokine profiles may produce diverse outcomes [25]. As shown in Figure 3A, the pANP-treated group also induced few Tregs versus the empty vector control, suggesting that TGF- $\beta$ may be involved in the process (Figure 2E \&2F). However, attenuation of NPRA signaling by pNP73-102 induced a greater amount of SOCS3 and generated more Tregs which supports the hypothesis that ANP signaling effects are mediated through production of tolerogenic DCs. Data show that ANP inhibits TGF- $\beta$-induced Smad2 and Smad3 nuclear translocation in rat pulmonary arterial smooth muscle cells [26], which is consistent with our finding that ANP induced less TGF- $\beta$ production in human DCs than NP73-102.

The SOCS3 protein was strongly induced by both IL- 6 and IL-10. SOCS3 selectively inhibits IL-6 signaling via its binding to the IL- 6 receptor, but does not inhibit the IL-10 receptor [27]. The suppressive effect of SOCS3 is primarily restricted to STAT3 [28], and these results show that pNP73-102 inhibits STAT3 activity and enhances SOCS3 expression. This is in marked contrast to pANP and the vector control, which induce STAT3 phosphorylation and decrease SOCS3 expression in $\mathrm{hmDCs}$. These data further support the idea that in this model, NPRA signaling in tolerogenic DCs involves the regulation of SOCS3 expression and STAT3 activity.

Cells exposed to pNP73-102 selectively diminished TLR2 expression compared to cells given pANP or vector. This could be explained by decreased NF- $\kappa \mathrm{B}-$ mediated down-regulation of TLR through a binding site for NF- $\kappa$ B on the TLR2 promoter [29]. Reports have indicated that down-regulation of both TLR4 and TLR2 expression in mice decreases the expression of inflammatory cytokines and enhances production of anti-inflammatory cytokines, which induce immune tolerance [30]. Significantly, enhanced MyD88 expression was found in DCs treated with pNP73-102 compared to pANP and vector control. An LPS-inducible MyD88 is defective in its ability to induce IRAK phosphorylation and behaves as a dominant-negative inhibitor of LPSinduced NF- $\kappa$ B activation [31]. Also, MyD88-knockout mice show significantly reduced expression of SOCS3 [32], which is in part consistent with our data, although it is unclear why pANP induced higher SOCS3 expression in the absence of MyD88 than vector control. Thus, the enhanced expression of both SOCS3 and MyD88 in DCs may be associated with a reduced response to ANP, whereas the specific enhancement of SOCS3 and/or MyD88 expression may explain the generation of tolerogenic DCs.

Indirect inhibition of JAKs due to the binding of SOCS to membrane proximal regions of receptor chains results in steric hindrance of constitutive JAK binding to the receptor [33]. Inhibition of NPRA signaling by pNP73-102 through SOCS3 might occur by this mechanism. The IP data support this hypothesis. NPRA binds to SOCS3, and this interaction might contribute to the effects of NPRA signaling on immunoregulation. NPRA also binds to TLR2 and STAT3. However, TLR2 and SOCS3 involvement in regulation of NPRA expression (unpublished data) might result from these protein interactions rather than STAT3 involvement. Further work is needed to clarify whether the interactions among these proteins are direct or indirect.

In our animal model, we found that NPRA-/- mice had decreased expression of Th2-like cytokines, and that adoptive transfer of DCs from WT to NPRA-/- mice restored levels of these cytokines to those seen in WT. This is an important finding since it complements the in vitro results in an animal model. The lungs from NPRA-/- mice given DCs from WT mice exhibited inflammation similar to that of the WT. OVA-treated NPRA-/- mice given no DCs or given DCs from NPRA-/- mice did not have significant lung inflammation (Figure 6A, B), suggesting that DCs are the key mediators in modulating lung inflammation by NPRA signaling.

Taken together, our results demonstrate a novel mechanism for integration of TLR2, STAT3 and SOCS3 with NPRA signaling to regulate the immunomodulatory activity of DCs. They support the hypothesis that inhibition of NPRA signaling and TLR2 expression in DCs induces more IL-10 and TGF- $\beta$ secretion and increases 
SOCS3 expression, thereby promoting the generation of Treg cells.

\section{Additional material}

Additional file 1: Additional file 1. This supplementary material contains additional information about the viability assay, the T cell suppression assay, the luciferase assay, and the experiments in mice.

\section{Acknowledgements}

SSM is supported by grants from the Veterans' Affairs Merit Review Award and U.S. National Institutes of Health \#5RO1HL71101-01A2. We thank Dr. Hirohide Yoshikawa (JFCR Cancer Institute, Japan) for his pSOCS3 constructs, Drs. G. J. Rosman and D. A. Miller (Biotechniques, Germany) for their gift of PSTAT3-YFP, Dr. Toshio Hirano (Osaka University, Japan) for the plasmid pSTAT3-Iuc, Dr. W. R. Gower, Jr. (University of South Florida, USA) for the plasmid, pNPRA, and Dr. Weidong Xu (University of South Florida, USA) for the plasmids, pANP and pNP73-102. We would also like to express our appreciation to Saneron-Cell Therapeutics (Tampa, Florida, USA) for the cord blood cells and to the Moffitt Flow Cytometry and Microscopy Core for their assistance.

\section{Author details}

'Department of Internal Medicine, Division of Allergy and Immunology, University of South Florida College of Medicine, Tampa, FL 33612, USA. ${ }^{2}$ Department of Molecular Medicine, University of South Florida College of Medicine, Tampa, FL 33612, USA. ${ }^{3}$ James A. Haley Veterans Hospital Medical Center, Tampa, FL 33612, USA. ${ }^{4}$ Department of Internal Medicine, Division of Endocrinology, University of South Florida College of Medicine, Tampa, FL 33612, USA. ${ }^{5}$ Donald A. Adam Comprehensive Melanoma Research Center, Moffitt Cancer Center, 12902 Magnolia Drive, SRB-24324, Tampa, FL 33612, USA. ${ }^{6}$ Department of Internal Medicine, Division of Translational Medicine, University of South Florida College of Medicine, Tampa, FL 33612, USA.

\section{Authors' contributions}

WZ: contributed equally to the acquisition and analysis of data. XC contributed equally to the acquisition of data. DC, WW, HY and XK provided technical support. JWW contributed to the discussion. SM reviewed the manuscript and data presentation. GH prepared and edited the manuscript. RFL reviewed the manuscript. SSM directed research, reviewed the manuscript. All authors have read and approved the final manuscript.

\section{Competing interests}

The authors declare that they have no competing interests.

Received: 23 April 2010 Accepted: 10 January 2011

Published: 10 January 2011

\section{References}

1. Passino C, Poletti R, Fontana M, Vergaro G, Prontera C, Gabutti A: Clinical relevance of non-cardiac determinants of natriuretic peptide levels. Clin Chem Lab Med 2008, 46:1515-23.

2. Mohapatra SS: Role of natriuretic peptide signaling in modulating asthma and inflammation. Can Physiol Pharmacol 2007, 85:754-9.

3. Piechota M, Banach M, Jacon A, Rysz J: Natriuretic peptides in cardiovascular diseases. Cell Mol Biol Lett 2008, 13:155-81.

4. Pandey KN: Internalization and trafficking of guanylyl cyclase/natriuretic peptide receptor-A. Peptide 2005, 26:985-1000.

5. Gutkowska J, Nemer M: Structure, expression and function of atrial natriuretic factor in extraatrial tissues. Endocrinol Rev 1989, 10:519-37.

6. Kiemer AK, Vollmar AM: Effects of different natriuretic peptides on nitric oxide synthesis in macrophages. Endocrinology 1997, 138:4282-90.

7. Morita R, Ukyo N, Furuya M, Uchiyama T, Hori T: Atrial natriuretic peptide polarizes human dendritic cells toward a Th2-promoting phenotype through its receptor guanylyl cyclase-coupled receptor A. J Immunol 2003, 15:5869-75.
8. Wang X, Xu W, Mohapatra S, Kong X, Li X, Lockey RF: Prevention of airway inflammation with topical cream containing imiquimod and small interfering RNA for natriuretic peptide receptor. Genet Vaccines Ther 2008, 15:6-7.

9. Kumar M, Kong X, Behera AK, Hellermann GR, Lockey RF, Mohapatra SS: Chitosan IFN-gamma-pDNA Nanoparticle (CIN) Therapy for Allergic Asthma. Genet Vaccines Ther 2003, 27:1-3.

10. Mohapatra SS, Lockey RF, Vesely DL, Gower WR Jr: Natriuretic peptides and genesis of asthma: an emerging paradigm? J Allergy Clin Immunol 2004, 114:520-26.

11. Suss $G$, Shortman $K$ : A subclass of dendritic cells kills CD4 T cells via Fas/ Fas-ligand-induced apoptosis. J Exp Med 1996, 183:1789-96.

12. Zheng Z, Narita M, Takahashi M, Liu A, Furukawa T, Toba K: Induction of $T$ cell anergy by the treatment with IL-10-treated dendritic cells. Comp Immunol Microbiol Infect Dis 2004, 27:93-103.

13. Jonuleit $\mathrm{H}$, Adema G, Schmitt E: Immune regulation by regulatory T cells: implications for transplantation. Transpl Immunol 2003, 11:267-76.

14. Akdis CA, Akdis M: Mechanisms and treatment of allergic disease in the big picture of regulatory T cells. J Allergy Clin Immunol 2009, 123:735-46.

15. Henry E, Desmet CJ, Garze V, Fievez L, Bedoret D, Heirman C: Dendritic cells genetically engineered to express IL-10 induce long-lasting antigen-specific tolerance in experimental asthma. J Immunol 2008, 181:7230-42.

16. Zhang W, Yang H, Kong X, Mohapatra S, San Juan-Vergara H, Hellermann G: Inhibition of respiratory syncytial virus infection with intranasal siRNA nanoparticles targeting the viral NS1 gene. Nat Med 2005, 11:56-62.

17. Zhao X, Ye F, Chen L, Lu W, Xie X: Human epithelial ovarian carcinoma cell-derived cytokines cooperatively induce activated CD4+CD25CD45RA+ naïve T cells to express forkhead box protein 3 and exhibit suppressive ability in vitro. Cancer Sci 2009, 100:2143-51.

18. Liu Y, Zhang W, Chan T, Saxena A, Xiang J: Engineered fusion hybrid vaccine of IL-4 gene-modified myeloma and relative mature dendritic cells enhances antitumor immunity. Leuk Res 2002, 26:757-63.

19. Zhang W, Chen Z, Li F, Kamencic H, Juurlink B, Gordon JR: Tumour necrosis factor-alpha (TNF-alpha) transgene-expressing dendritic cells (DCs) undergo augmented cellular maturation and induce more robust T-cell activation and anti-tumour immunity than DCs generated in recombinant TNF-alpha. Immunology 2003, 108:177-88.

20. Sommer K, Njau F, Wittkop U, Thalmann J, Bartling G, Wagner A: Identification of high- and low-virulent strains of Chlamydia pneumoniae by their characterization in a mouse pneumonia model. FEMS Immunol Med Microbiol 2009, 55:206-14.

21. Dalpke AH, Eckerle S, Frey M, Heeg K: Triggering of Toll-like receptors modulates IFN-gamma signaling: involvement of serine 727 STAT1 phosphorylation and suppressors of cytokine signaling. Eur I Immunol 2003, 33:1776-87.

22. Iruretagoyena MI, Sepulveda SE, Lezana JP, Hermoso M, Bronfman M, Gutierrez MA: Inhibition of nuclear factor-kappa B enhances the capacity of immature dendritic cells to induce antigen-specific tolerance in experimental autoimmune encephalomyelitis. J Pharmacol Exp Ther 2006, 318:59-67.

23. Bettelli E, Carrier Y, Gao W, Korn T, Strom TB, Oukka M: Reciprocal developmental pathways for the generation of pathogenic effector TH17 and regulatory T cells. Nature 2006, 441:235-38.

24. Toh ML, Miossec P: The role of T cells in rheumatoid arthritis: new subsets and new targets. Curr Opin Rheumatol 2007, 19:284-88.

25. Zhou L, Lopes JE, Chong MM, Ivanov II, Min R, Victora GD: TGF- $\beta$-induced Foxp3 inhibits Th17 cell differentiation by antagonizing RORyt function. Nature 2008, 453:236-40.

26. Li P, Oparil S, Novak L, Cao X, Shi W, Lucas J: ANP signaling inhibits TGF- $\beta$ induced Smad2 and Smad3 unclear translocation and extracellular matrix expression in rat pulmonary arterial smooth muscle cells. J Appl Physiol 2007, 102:390-98.

27. Yasukawa H, Ohishi M, Mori H, Murakami M, Chinen T, Aki D: IL-6 induces an anti-inflammatory response in the absence of SOCS3 in macrophages. Nat Immunol 2003, 4:551-56.

28. Howard JK, Cave BJ, Oksanen LJ, Tzameli I, Biorbaek C, Flier JS: Enhanced leptin sensitivity and attenuation of diet-induced obesity in mice with haploinsufficiency of Socs3. Nat Med 2004, 10:734-38.

29. Musikacharoen T, Matsuguchi T, Kikuchi T, Yoshikai Y: NF-kB and STAT5 play important roles in the regulation of mouse Toll-like receptor 2 gene expressions. J Immunol 2001, 166:4516-24. 
30. Eguchi M, Sekiya Y, Suzuki M, Yamamoto T, Matsui H: An oral salmonella vaccine promotes the down-regulation of cell surface Toll-like receptor 4 (TLR4) and TLR2 expression in mice. FEMS Immunol Med Microbiol 2007, 50:300-8.

31. Janssens S, Burns K, Tschopp J, Beyaert R: Regulation of interleukin-1- and lipopolysaccharide-induced NF-kappaB activation by alternative splicing of MyD88. Curr Biol 2002, 12:467-71.

32. Campbell JS, Riehle K, Brooling JT, Bauer RL, Mitchell C, Fausto N: Proinflammatory cytokine production in liver regeneration is MyD88dependent, but independent of Cd14, TIr2, and TIr4. J Immunol 2006, 176:2522-28.

33. Ilangumaran $\mathrm{S}$, Ramanathan $\mathrm{S}$, Rottapel R: Regulation of the immune system by SOCS family adaptor proteins. Seminars Immunol 2004, 16:351-65.

doi:10.1186/1479-0556-9-3

Cite this article as: Zhang et al.: Plasmid-encoded NP73-102 modulates atrial natriuretic peptide receptor signaling and plays a critical role in inducing tolerogenic dendritic cells. Genetic Vaccines and Therapy 2011 9:3.

\section{Submit your next manuscript to BioMed Central} and take full advantage of:

- Convenient online submission

- Thorough peer review

- No space constraints or color figure charges

- Immediate publication on acceptance

- Inclusion in PubMed, CAS, Scopus and Google Scholar

- Research which is freely available for redistribution

Submit your manuscript at www.biomedcentral.com/submit 